\title{
Establishing Analytical Validity of BeadChip Array Genotype Data by Comparison to Whole-Genome Sequence and Standard Benchmark Datasets
}

Praveen F Cherukuri ( $\nabla$ praveen.cherukuri@sanfordhealth.org )

Sanford Health

Melissa M. Soe

Sanford Health

David E. Condon

Sanford Health

Shubhi Bartaria

Sanford Health

Kaitlynn Meis

Sanford Health

Shaopeng Gu

Sanford Health

Frederick G. Frost

Sanford Health

Lindsay M. Fricke

Sanford Health

Krzysztof P. Lubieniecki

Sanford Health

Joanna M. Lubieniecka

Sanford Health

Robert E. Pyatt

Sanford Health

Catherine Hajek

Sanford Health

Cornelius F. Boerkoel

Sanford Health

Lynn Carmichael

Sanford Health 
Keywords: Clinical genotyping, genotyping error, analytical validation

Posted Date: July 28th, 2021

DOI: https://doi.org/10.21203/rs.3.rs-745072/v1

License: (c) (1) This work is licensed under a Creative Commons Attribution 4.0 International License. Read Full License

Version of Record: A version of this preprint was published at BMC Medical Genomics on March 14th, 2022. See the published version at https://doi.org/10.1186/s12920-022-01199-8. 
1 Establishing analytical validity of BeadChip array genotype data by

2 comparison to whole-genome sequence and standard benchmark datasets

4 Praveen F. Cherukuri ${ }^{1,2,3}$, Melissa M. Soe ${ }^{1}$, David E. Condon ${ }^{1,2}$, Shubhi Bartaria ${ }^{1}$, Kaitlynn 5 Meis $^{1}$, Shaopeng $\mathrm{Gu}^{1}$, Frederick G. Frost ${ }^{1}$, Lindsay M. Fricke ${ }^{1}$, Krzysztof P. Lubieniecki ${ }^{1,2,3}$, 6 Joanna M. Lubieniecka ${ }^{1,2,3}$, Robert E. Pyatt ${ }^{1,2}$, Catherine Hajek ${ }^{1,2}$, Cornelius F. Boerkoel ${ }^{1}$, Lynn Carmichael $^{1}$

\section{Correspondence:}

18 Praveen F. Cherukuri, $\mathrm{PhD}$

19 Imagenetics, Sanford Health

$201410 \mathrm{~W} 25^{\text {th }}$ St. Room \#302

21 Sioux Falls, SD 57105

22 Phone: +1 (605) 404-4265

23 Fax: N/A

24 Email: praveen.cherukuri@ sanfordhealth.org 
Abstract

26 Background Clinical use of genotype data requires high positive predictive value (PPV) and

27 thorough understanding of the genotyping platform characteristics. BeadChip arrays, such as the

28 Global Screening Array (GSA), potentially offer a high-throughput, low-cost clinical screen for

29 known variants. We hypothesize that quality assessment and comparison to whole-genome

30 sequence and benchmark data establish the analytical validity of GSA genotyping.

31 Methods To test this hypothesis, we selected 263 samples from Coriell, generated GSA

32 genotypes in triplicate, generated whole genome sequence (rWGS) genotypes, assessed the

33 quality of each set of genotypes, and compared each set of genotypes to each other and to the

341000 Genomes Phase 3 (1KG) genotypes, a performance benchmark. For 59 genes (MAP59), we

35 also performed theoretical and empirical evaluation of variants deemed medically actionable

36 predispositions.

37 Results Quality analyses detected sample contamination and increased assay failure along the

38 chip margins. Comparison to benchmark data demonstrated that $>82 \%$ of the GSA assays had a

39 PPV of 1. GSA assays targeting transitions, genomic regions of high complexity, and common

40 variants performed better than those targeting transversions, regions of low complexity, and rare

41 variants. Comparison of GSA data to rWGS and $1 \mathrm{KG}$ data showed $>99.3 \%$ concordance across

42 all measured parameters. GSA detection of variation within the MAP59 genes was 3/261

43 consistent with predictions from prior studies.

44 Conclusion We establish the analytical validity of GSA assays using quality analytics and

45 comparison to benchmark and rWGS data. GSA assays meet the standards of a clinical screen

46 although assays interrogating rare variants, transversions, and variants within low-complexity

47 regions require careful evaluation. 
49 Keywords Clinical genotyping, genotyping error, analytical validation

\section{Background}

52 Clinical genotyping requires assays with high positive predictive value (PPV) and minimal error

53 (1). The impact of genotyping error has been observed for variant association tests (2), sibling-

54 pair analyses (3), and variant and genotype interpretation (4). Genotyping errors occur when the

55 observed genotype does not correspond to an individual's true genotype (5). Such errors arise

56 from multiple factors including, but not limited to, biases in modeling algorithms (6), sample and

57 technical batch effects (7), paralogous genomic regions (8), sample contamination (9), allele

58 frequency differences on genotyping platforms (10), and DNA sample quality (11).

60 Several methods have been developed to detect and minimize genotyping errors. These include

61 the quality control (QC) metrics of genotype call rate $(12,13)$ and sample contamination

62 detection (14). Additional methods include assessing departure from Hardy-Weinberg

63 Equilibrium (HWE) (15-17), information content for each chromosome before and after removal

64 of SNPs with high linkage disequilibrium (LD) (18), likelihood of error (19), departure from

65 expected Mendelian inheritance (4), and pedigree information (20).

66

67 QC of genotype data minimizes the likelihood of errors (11,21, 22). Estimating true genotypes

68 and detecting errors require well-characterized benchmark datasets such as those described for

69 bioinformatic genotyping pipelines (23), quality control algorithms (24), and sequencing

70 platforms (25-27). Additionally, theoretical benchmark datasets are needed for analysis of 
71 genotype data and estimating genotyping error (28). Compared to NGS $(26,29,30)$, genotyping

72 via DNA hybridization has distinct, well described genotyping and platform biases $(10,31,32)$.

73

74 Clinical genotyping using DNA hybridization, e.g., the Global Screening Array (GSA), requires

75 a comprehensive analytical framework to detect and limit error. Based on current research

76 methodologies, we propose analytical validation of GSA genotyping by assessment of quality

77 metrics and by comparison to truth sets, namely, those of the 1000 Genomes Phase 3 (1KG), the

78 National Institute of Standards and Technology (NIST), and the Genome in a Bottle Consortium

79 (GiAB). To test this, we selected 263 Coriell DNA samples and, for each sample, generated

80 whole genome sequence ( $\mathrm{rWGS}$ ) at $>37 \mathrm{x}$ read depth and GSA genotypes in triplicate. These data

81 were compared to each other and to the corresponding publicly available truth sets. Additionally,

82 we characterized each GSA assay performance and biases by stratifying GSA assays according

83 to allele frequency, nucleotide variant class, low-complexity regions, medically actionable

84 variants, and other genomic features.

85 
Methods

\section{$87 \quad$ Aim and design of study}

88 This study defines an analytical validation framework for detecting and limiting genotyping error

89 in GSA data (Figure 1). To minimize platform specific genotyping biases, internally generated

90 genotype data from independent platforms were paired and compared with publicly available

91 genotype datasets.

\section{Samples and datasets}

94 To generate a reference genotype cluster file for the GSA, 664 DNA samples were purchased

95 from the Coriell Institute for Medical Research, Camden, NJ, and 460 samples were selected

96 from the Sanford Biobank. These samples were selected to cover different ethnicities (14 Coriell

97 diversity panels) and the technical variability of the DNA extraction methods (460 samples from

98 the Sanford Biobank). To capture the technical variability of the Infinium ${ }^{\circledR}$ HTS Assay protocol

99 (Illumina Inc.), all samples were genotyped in triplicate (by different technicians, robot-

100 instrument configurations, reagent lots, and days) using the Infinium Global Screening Array-24

101 v.1.0 BeadChip. The resulting data were loaded into GenomeStudio v2.0.2 and used to generate

102 the genotype cluster file per manufacturer recommendations

103 (https://www.illumina.com/Documents/products/technotes/technote_infinium_genotyping_data_

104 analysis.pdf). Of the 1,104 samples used in cluster file generation 72 were also included among

105 the 263 samples used to define analytical validity (see below and Supplementary Materials -1

106 and 2). Two hundred sixty-three (263) DNA samples from Coriell (https://www.coriell.org) were

107 selected as representative of individuals from the 1000 Genomes Project Consortium $(n=258)$

108 and from the Genome in a Bottle Consortium (GiAB) $(33)(n=5)$. Additionally, they were 
109 selected to assess assays genotyping alleles with $\geq 1 \%$ minor allele frequency (MAF) in the

110 general population (Supplementary Table S1). These 263 DNA samples were resequenced with

111 whole genome sequencing (rWGS) and genotyped in triplicate ( $263 \times 3)$ with the GSA. These

112 data were compared to $1 \mathrm{KG}$ and to publicly available Whole Genome Sequence (pWGS) data

113 (1KG phase 3; downloaded: June 2018). This defined 4 genotype datasets for the 263 samples:

114 (i) triplicate GSA genotypes (ii) pWGS, (iii) rWGS, and (iv) $1 \mathrm{KG}$ (Supplementary Table S2).

116 Human Genome reference sequence

117 Mapping, alignment, and genotyping were performed using Human Reference Sequence

118 GRCh37 (Genome Reference Consortium Human build 37).

120 Data generation

\section{Illumina Infinium GSA}

122 Illumina's GSA - 24 v1.0 BeadChips (24-sample format) were processed following the standard

123 Infinium High-throughput Screening (HTS) protocol using the Freedom EVO ${ }^{\circledR}$ platform (Tecan)

124 and AutoLoader 2.x (Illumina, Inc.). Raw intensity data for each bead on a BeadChip were

125 collected using the iScan ${ }^{\circledR}$ System (Illumina, Inc) and saved as intensity (*.idat) files. The

126 intensity files were converted to genotypes by the AutoConvert feature in the iScan Control

127 software using the GenCall algorithm and the Illumina GSA manifest (alleles labeled as A and

128 B) file. The normalized genotype data were saved as binary files (*.gtc) and used as input for

129 GenomeStudio v2.0.2 to generate preliminary Quality Control (QC) parameters (CallRate, 130 p10GC), B-allele frequency files, log-likelihood files, and Variant Call Format (VCF) files

131 (https://samtools.github.io/hts-specs/VCFv4.1.pdf). Genotypes were called relative to GRCh37 
132 using gtc_to_vcf.py (v1.1.1) (GitHub link: https://github.com/Illumina/GTCtoVCF). Alleles

133 matching the reference allele were encoded as ' 0 ', first alternate allele as ' 1 ', second alternate

134 allele as ' 2 ', and third alternate allele as ' 3 '. The allelic combinations for genotypes were

135 encoded as $0 / 0,0 / 1,1 / 1,0 / 2$, etc. for a total of 10 possible genotypes. All possible genotypes and 136 their comparisons are shown in Table 1.

\begin{tabular}{|c|c|c|c|c|c|c|c|c|c|c|c|c|c|c|c|c|}
\hline & \multicolumn{16}{|c|}{ Test Genotypes } \\
\hline \multirow{16}{*}{$\begin{array}{c}\text { True } \\
\text { Genotypes }\end{array}$} & - &. &.$/ 0$ &.$/ 1$ &.$/ 2$ &.$/ 3$ & $0 / 0$ & $0 / 1$ & $0 / 2$ & $0 / 3$ & $1 / 1$ & $1 / 2$ & $1 / 3$ & $2 / 2$ & $2 / 3$ & $3 / 3$ \\
\hline &. & na & $n a$ & na & na & $n a$ & na & na & na & na & na & na & $n a$ & $n a$ & na & na \\
\hline &.$/ 0$ & $n a$ & $t$ & $f$ & $f$ & $f$ & $f$ & $f$ & $f$ & $f$ & $f$ & $f$ & $f$ & $f$ & $f$ & $f$ \\
\hline &.$/ 1$ & na & $f$ & $t$ & $f$ & $f$ & $f$ & $f$ & $f$ & $f$ & $f$ & $f$ & $f$ & $f$ & $f$ & $f$ \\
\hline &.$/ 2$ & na & $f$ & $f$ & $t$ & $f$ & $f$ & $f$ & $f$ & $f$ & $f$ & $f$ & $f$ & $f$ & $f$ & $f$ \\
\hline &.$/ 3$ & na & $f$ & $f$ & $f$ & $t$ & $f$ & $f$ & $f$ & $f$ & $f$ & $f$ & $f$ & $f$ & $f$ & $f$ \\
\hline & $0 / 0$ & $n a$ & $f$ & $f$ & $f$ & $f$ & tn & $f p$ & $f p$ & $f p$ & $f p$ & $f p$ & $f p$ & $f p$ & $f p$ & $f p$ \\
\hline & $0 / 1$ & na & $f$ & $f$ & $f$ & $f$ & $f n$ & $t p$ & $x$ & $x$ & $x$ & $x$ & $x$ & $x$ & $x$ & $x$ \\
\hline & $0 / 2$ & $n a$ & $f$ & $f$ & $f$ & $f$ & $f n$ & $x$ & $t p$ & $x$ & $x$ & $x$ & $x$ & $x$ & $x$ & $x$ \\
\hline & $0 / 3$ & $n a$ & $f$ & $f$ & $f$ & $f$ & $f n$ & $x$ & $x$ & $t p$ & $x$ & $x$ & $x$ & $x$ & $x$ & $x$ \\
\hline & $1 / 1$ & $n a$ & $f$ & $f$ & $f$ & $f$ & $f n$ & $x$ & $x$ & $x$ & $t p$ & $x$ & $x$ & $x$ & $x$ & $x$ \\
\hline & $1 / 2$ & $n a$ & $f$ & $f$ & $f$ & $f$ & $f n$ & $x$ & $x$ & $x$ & $x$ & $t p$ & $x$ & $x$ & $x$ & $x$ \\
\hline & $1 / 3$ & $n a$ & $f$ & $f$ & $f$ & $f$ & $f n$ & $x$ & $x$ & $x$ & $x$ & $x$ & $t p$ & $x$ & $x$ & $x$ \\
\hline & $2 / 2$ & $n a$ & $f$ & $f$ & $f$ & $f$ & $f n$ & $x$ & $x$ & $x$ & $x$ & $x$ & $x$ & $t p$ & $x$ & $x$ \\
\hline & $2 / 3$ & na & $f$ & $f$ & $f$ & $f$ & $f n$ & $x$ & $x$ & $x$ & $x$ & $x$ & $x$ & $x$ & $t p$ & $x$ \\
\hline & $3 / 3$ & $n a$ & $f$ & $f$ & $f$ & $f$ & $f n$ & $x$ & $x$ & $x$ & $x$ & $x$ & $x$ & $x$ & $x$ & $t p$ \\
\hline
\end{tabular}

Abbreviations: $t p$, true positive; fp, false positive; tn, true negative; fn, false negative; $x$, other discordant genotypes; $n a$, no data; $f$, false genotype; $t$, true genotype.

\section{Whole genome sequencing (rWGS)}

145 The 263 DNA validation samples purchased from Coriell were sequenced using the Illumina

146 HiSeqX by Genome.One (Sydney, Australia). rWGS produced an average of 731 million $150 \mathrm{bp}$

147 paired-end reads to give an average of $37 x$ depth of coverage (range: $32 x-42 x$ ) across the 
148 Human Genome (GRCh37) (Supplementary Tables S3, S4 and S5). Raw sequence data (fastq

149 files) were transferred to GenomeNext (http://genomenext.com) and processed using the

150 Churchill pipeline (34). QC data and genotypes (GRCh37) were saved as VCF, genomic VCF

151 (gVCF), and binary alignment (BAM) files. In total, 22.3 TB of rWGS data were archived on

152 Amazon Web Services Storage 3 (AWS S3).

153

154 Data processing

155 GSA quality control (QC)

156 Laboratory QC

157 Genotype clusters for the variants used for clinical reporting were manually curated to ensure

158 accurate variant calling. Other variants were automatically curated using Illumina-recommended

159 filters (Illumina’s technical Note:

160 https://www.illumina.com/Documents/products/technotes/technote_infinium_genotyping_data_a

161 nalysis.pdf). Using the data of DNA samples from 1,104 individuals run on the GSA in triplicate,

162 the cluster file analyses of each GSA assay found that 610,771 (92\%) assays passed and 50,355

$163(8 \%)$ assays failed clustering quality control. Those that failed were excluded and marked as no-

164 calls (./.) in the VCF files.

165

166 Bioinformatics QC

167 The GSA data ( $\mathrm{n}=263 \times 3$ replicates) were stratified by the GSA BeadChip and the sample

168 location on the BeadChip (row, column) and grouped by sample replicate. For each sample, the

169610,771 assays that passed cluster file QC were used to evaluate the following parameters: (i)

170 genotype call rate, (ii) p10GC, and (iii) estimated sample contamination. Using in-house code, 
171 sample contamination was estimated according to the method of Jun, G. and colleagues (Jun et

172 al. 2012) (Methodology is described in Supplementary Material Section 5). Aggregate QC

173 analyses are shown in Figure 2.

174

\section{Data comparisons}

\section{Principal component analysis}

177 Principal component analysis (PCA), a tool commonly used in genotyping studies $(35,36)$ to

178 reduce multiple dimensions in order to synthesize and summarize the main structural

179 components, was used to test for intact super-population structure as a corollary for absence of 180 batch and technical artifacts in the genotyping datasets. PCA structure derived from GSA data 181 was compared to the super-population structure derived from $1 \mathrm{KG}$ data.

\section{Whole Genome Sequence data quality control (QC)}

\section{Bioinformatics QC}

185 For bioinformatics quality control of rWGS data $(n=263)$, central tendency and anomalous

186 outlier data points were assessed for (i) total processed reads, (ii) discordant reads, (iii) mapq0

187 reads, (iv) unmapped reads, (v) mapped reads, and (vi) average depth of sequencing

188 (Supplementary Tables S3 and S4). On average $>95 \%$ of processed reads per sample

$189(731,227,993 / 767,540,183$ reads $)$ mapped to the reference sequence. Because the concordance 190 of two rWGS datasets (HG00111 and HG00257) with the $1 \mathrm{KG}$ data were 0.870 and 0.622 , they 191 were dropped from our GSA analyses leaving a total of 261 samples in the rWGS dataset.

\section{Performance metrics}




\section{Genotype concordance, Sensitivity, Specificity and Positive Predictive Value (PPV)}

195 GSA and rWGS genotypes were compared to each other and to $1 \mathrm{KG}$ genotypes using the

196 following performance metrics: (i) genotype concordance (C), (ii) sensitivity (S), (iii) specificity

197 (P), and (iv) positive predictive value (PPV). We used the following definitions of genotype

198 classification to label genotypes as positive [true positive ( $t p)$, false positive $(f p)$ ], negative [true

199 negative $(t n)$, false negative $(f n)$ ], or discordant $(x)$ (Table 1):

200

$$
\begin{aligned}
& a=\sum t p . \\
& b=\sum f p . \\
& c=\sum t n . \\
& d=\sum f n \\
& z=\sum x .
\end{aligned}
$$

206 Given the above definitions of true / false positive and negative and discordant genotypes (see

207 Table 1), we computed the performance metrics as follows:

208

209 Genotype concordance (C)

210

$$
C=\left(\frac{a+c}{a+b+c+d+z}\right)
$$

211 Sensitivity (S)

$$
S=\left(\frac{a}{a+d}\right)
$$

213 Specificity $(\mathrm{P})$

$$
P=\left(\frac{c}{c+b}\right)
$$

215 Positive predictive value (PPV) 


$$
P P V=\left(\frac{a}{a+b}\right)
$$

\section{Classification of GSA assays}

$220 \quad$ Variation type

221 GSA assays were stratified according to variant classes: single nucleotide variants (SNVs;

222 656,601), multi-allelic variants (MAVs; 616), deletions (DEL; 2,799), and insertions (INS;

$2231,110)$.

224

\section{Nucleotide change class}

226 By parsing the VCF files and cataloging the alternate nucleotide, SNVs were stratified by

227 whether the nucleotide change was a transition or a transversion.

228

229 Allele frequency

230 SNVs were binned into 13 strata based on the alternate allele frequency reported in the $1 \mathrm{KG}$

231 VCF file (allele frequency * 100): (a) [0 - 0.1\%], (b) (0.1-1\%], (c) (1-5\%], (d) (5-10\%], (e) (10-

232 20\%], (f) (20-30\%], (g) (30-40\%], (h) (40-50\%], (i) (50-60\%], (j) (60-70\%], (k) (70-80\%], (1)

233 (80-90\%], and (m) (90-100\%].

234

235 Genomic complexity of variation locus (low-complexity regions)

236 To categorize SNVs based on the genomic complexity of the GSA assay locus, we used the

237 UCSC genome browser bed-file definitions to define simple-repeats, micro-satellite regions, and 238 low-complexity regions. The SimpRep, Microsatellites, and RepeatMasker bedfiles were 
239 downloaded from the UCSC Genome Browser FTP site and intersected with the GSA manifest

240 file. Across the GRCh37 reference sequence, there were 962,715 simple repeat, 41,573

241 microsatellite, and 5,298,131 RepeatMasker regions.

243 GSA panels

\section{Medically Actionable Predispositions (MAP) 59 gene panel}

245 GSA assays targeting potentially disease-associated variants in MAP59 genes (37) were selected

246 in a multistep process (Table 2). Firstly, GSA assays that interrogated positions within 1000-

247 bases upstream and downstream of the transcript start and end in Human Genome build GRCh37

248 were selected for the RefSeq transcript chosen for each gene. Secondly, alleles were annotated

249 with their respective ClinVar classifications, and those that had at least one classification of

250 pathogenic or likely pathogenic were selected. Thirdly, these assays were curated by clinical and

251 laboratory staff to define a managed variant list (MVL) of 1,883 assays appropriate for clinical

252 reporting.

Table 2. Selection process for GSA assays targeting genotypes considered Medically Actionable Predispositions

\begin{tabular}{|l|r|}
\hline \multicolumn{1}{|c|}{ GSA MAP59 subsets } & $\begin{array}{c}\text { Number of } \\
\text { Assays }\end{array}$ \\
\hline GSA MAP59 (+/- 1kb) & 6,841 \\
\hline GSA MAP59 (select: “ClinVar" AND “Predicted Path”) & 5,075 \\
\hline $\begin{array}{l}\text { GSA MAP59 (select: “ClinVar" AND "Predicted Path” AND } \\
\text { "HGMD”) }\end{array}$ & 3,082 \\
\hline $\begin{array}{l}\text { GSA MAP59 MVL (select: “ClinVar" AND “Predicted Path” AND } \\
\text { "HGMD” AND “Curated") }\end{array}$ & 1,883 \\
\hline
\end{tabular}




\section{Statistics and compute infrastructure}

263 Statistical analyses and data visualization were performed using R (version 3.4.3). Data analysis

264 was done on a Linux Operating System with the following configuration: x86_64, 32 CPUs, 2.8

265 GHz AMD Opteron Processor 6320. AWS EC2 instances were spun-up for large compute jobs.

266 All NGS and GSA data were archived on AWS S3. In-house software and data processing code

267 and scripts were written primarily in Perl, Ruby, awk, and bash. 


\section{Data summary}

271 DNA samples from 263 individuals were purchased from Coriell and genotyped in triplicate

$272(\mathrm{n}=789)$ with the GSA. Genotypes and data for each replicate were saved to a VCF file. The

273 GSA data were grouped and summarized as replicate datasets 1, 2, and 3. Of the 263 samples,

274258 were present in the $1 \mathrm{KG}$. Of the other 5 samples, 3 were from the Personal Genomes Project

275 (PGP) (38), and 2 were from the NIGMS Human Genetic Cell Repository. The 263 x 3 data

276 were compared with the $1 \mathrm{KG}$ data and with two WGS datasets, the resequenced WGS data $(\mathrm{n}=$

$277261 ;$ rWGS = 37x $)$, and the downloaded public WGS data $(\mathrm{n}=24 ; \mathrm{pWGS}=51 \mathrm{x})$.

279 Principal component analysis defines the same population structure in GSA data and 1KG

280 data

281 Principal component analysis (PCA) on each replicate of autosomal GSA data identified 5 major

282 super populations conserved across replicates (Supplementary Material - Section 4). PCA

283 analysis of the $1 \mathrm{KG}$ autosomal genotype data from the same loci generated a similar population

284 structure (Figure 2A). This suggested that the GSA data did not have confounding technical

285 factors skewing the PCA plot. To determine if fewer GSA genotypes were sufficient for this test, 286 we randomly sub-sampled close to 10,000 genotypes; these recapitulated the population structure 287 (Supplementary Material - Section 4). 

detectable stochastic QC failure

292 Given that PCA did not detect major technical confounders within the GSA genotypes, we 293 analyzed the $263 \times 3$ data for quality and reproducibility (10) (Table 3 and Figure 3). These data 294 were stratified by BeadChip identifiers and sample location on the BeadChip (row, column). 295 Additionally, samples were grouped by replicates, and each replicate sample was evaluated for (i) genotype call-rate ( $\mathrm{n}=610,771$ assays), (ii) p10GC, and (iii) estimated DNA sample contamination. Aggregate quality control analysis showed a lower p10GC in higher numbered rows on the BeadChip (Figure 2B); excluding contaminated samples, p10GC ranged from 0.56$0.61($ Mean $=0.60, \mathrm{SD}=0.0085)$ in row 1 and from 0.50-0.61 $($ Mean $=0.55, \mathrm{SD}=0.03)$ in row 12. Over $99 \%$ (782 / 789) of samples had a call rate of $>0.98 .3$ samples in the third replicate dataset were contaminated, and 2 of these 3 samples had a call rate $<0.98(0.93$ and 0.94 , Figure

302 2C).

Table 3. Summary of GSA triplicate data and average number of genotypes detected in all 304 triplicate samples.

\begin{tabular}{|l|r|r|r|r|}
\hline & \multicolumn{1}{|c|}{ Replicate 1 } & \multicolumn{1}{c|}{ Replicate 2 } & \multicolumn{1}{c|}{ Replicate 3 } & \multicolumn{1}{c|}{ All data } \\
\hline Total Genotypes called* & $609,852( \pm 1,625)$ & $609,723( \pm 2,548)$ & $609,648( \pm 3,501)$ & $609,741( \pm 2,668)$ \\
\hline Missing Genotypes & $919( \pm 1,625)$ & $1,048( \pm 2,548)$ & $1,122( \pm 3,501)$ & $1,030( \pm 2,668)$ \\
\hline Autosomal Genotypes & $599,666( \pm 1,619)$ & $599,538( \pm 2,538)$ & $599,467( \pm 3,459)$ & $599,557( \pm 2,645)$ \\
\hline $\begin{array}{l}\text { Autosomal Heterozygous } \\
\text { Genotypes }\end{array}$ & $103,328( \pm 4,061)$ & $103,221( \pm 4,063)$ & $103,309( \pm 4,087)$ & $103,286( \pm 4,066)$ \\
\hline $\begin{array}{l}\text { Autosomal Homozygous } \\
\text { Alternate Genotypes }\end{array}$ & $60,652( \pm 3,081)$ & $60,643( \pm 3,098)$ & $60,623( \pm 3,081)$ & $60,639( \pm 3,083)$ \\
\hline
\end{tabular}

${ }^{a}$ We define the alternate genotype as a genotype different from the hg19 reference genotype.

307 To test if call-rates were reproducible across replicates, we measured deviations from 308 expectation and dispersion. The first approach, a Z-score method, computes the number of 309 standard deviations a replicate sample call-rate is from the expected as defined by the global 310 dataset average and standard deviation. The second approach computes the average call-rate of 
311 all replicates for a given sample and then computes variation around the average. Using the Z-

312 score method, 7 samples had a Z-score $\leq-4$. With a more conservative cut-off (Z-score $<-3$ ), 11

313 samples deviated from expectation (Supplementary Figure 12). When analyzed relative to the

314 BeadChip row and column, outlier Z-scores occurred for wells on the edge of the Illumina

315 BeadChip - R12C01 or R11C01; the only exceptions were two contaminated samples that were

316 in wells R01C01 and R01C02. Dispersion metrics calculated for call rates across each set of

317 three replicates (Table 4) identified higher relative dispersion for the same samples detected by

318 the Z-score method.

Table 4. Dispersion data paired with Z-score data

\begin{tabular}{|c|c|c|c|c|c|c|c|}
\hline Sample & R1 call rate & R2 call rate & R3 call rate & Average & $\begin{array}{c}\text { Z-score } \\
\text { detected } \\
\text { replicate }\end{array}$ & $\begin{array}{c}\text { Dispersion } \\
\text { (call rate) }\end{array}$ & $\begin{array}{c}\text { Estimated } \\
\text { Contamination }\end{array}$ \\
\hline NA20351 & 0.9987 & 0.9544 & 0.9989 & 0.984 & R2 & 0.000447 & 2.75 \\
\hline NA19475 & 0.9793 & 0.9856 & 0.9961 & 0.987 & R1 & 0.000048 & 2.5 \\
\hline NA19472 & 0.9986 & 0.9993 & 0.9451 & 0.981 & R3 & 0.000659 & 3.75 \\
\hline NA19390 & 0.9918 & 0.9706 & 0.9989 & 0.987 & R2 & 0.000146 & 2.5 \\
\hline NA18861 & 0.9991 & 0.9707 & 0.9817 & 0.984 & R2 & 0.000139 & 2.5 \\
\hline NA18508 & 0.9987 & 0.9988 & 0.9318 & 0.976 & R3 & 0.001021 & 4 \\
\hline HG03279 & 0.9793 & 0.9878 & 0.9967 & 0.988 & R1 & 0.000051 & 2.5 \\
\hline NA19466 & 0.9989 & 0.9988 & 0.9971 & 0.998 & - & 0.000001 & $4.25(\mathrm{R} 3)$ \\
\hline
\end{tabular}

323 Replicate pairwise concordance was calculated to assess the stochastic nature of sample

324 genotyping quality and these were plotted as a 3D scatter-plot: [R1 vs. R2 (x-axis), R2 vs. R3 (y-

325 axis), and R1 vs. R3 (z-axis)] (Figure 3). The data along the diagonal of the cube are correlated

326 data values across triplicates for all measured GSA genotypes for a given DNA sample. 260 of

327263 samples in the triplicate dataset (262 / 263 R1 vs. R2; 260 / 263 R2 vs. R3; 261 / 263 R1 vs.

328 R3) had concordance greater than 0.999 between replicates suggesting high reproducibility. Off- 
329 diagonal points, i.e., those with poor call rates $(<0.98)$ (Figure $2 \mathrm{~B})$, were along the edge of

330 Illumina chip or contaminated; we did not observe random occurrence of poor call rates.

332 Grouping GSA assays by variation type shows that SNVs have $>0.99$ performance relative

\section{3 to the benchmark dataset $1 \mathrm{KG}$ across all metrics.}

334 Of the 263 samples with GSA data, 258 had corresponding $1 \mathrm{KG}$ genotype data for computing 335 performance metrics of concordance, sensitivity, specificity, and PPV. Each GSA assay was 336 grouped according to the type of nucleotide change assessed: (a) single nucleotide variant

337 (SNV), (b) multi-allelic variant (MAV), (c) insertion, and (d) deletion (Table 5). SNVs

338 accounted for $99.3 \%(656,601 / 661,126) ; 610,771$ of these passed cluster file quality control,

339 and 594,361 detected genotypes present in the 1KG. Among the MAV assays, 526 of 616 passed

340 cluster file QC; however, because only 3 of these had genotypes present in the $1 \mathrm{KG}$, we

341 excluded MAVs from further analysis. Among insertion assays, 1,044 of 1,110 passed cluster

342 file QC, and 36 of these had genotypes present in the 1KG. Among deletion assays 2,677 of

3432,799 assays passed cluster file QC, and 95 of these had genotypes present in the1KG. Using the

344 three replicate GSA genotype datasets, the performance metrics of SNV assays were $>0.99$. In

345 contrast, insertion assays had highly variable concordance with the $1 \mathrm{KG}$, and deletion assays had

346 poor performance metrics (Figure 4A).

Table 5. Summary of GSA assays subgrouped by nucleotide variation type

\begin{tabular}{|l|r|r|r|}
\hline $\begin{array}{l}\text { Nucleotide variant type assay } \\
\text { subsets }\end{array}$ & $\begin{array}{c}\text { All GSA } \\
\text { data }\end{array}$ & $\begin{array}{c}\text { GSA pass } \\
\text { manifest } \\
\text { clusterfile } \\
\text { QC }\end{array}$ & $\begin{array}{c}\text { GSA pass } \\
\text { manifest QC } \\
\text { and present } \\
\text { in 1KG } \\
\text { Phase 3 }\end{array}$ \\
\hline Single Nucleotide Variants (SNVs) & 656,601 & 606,524 & 594,230 \\
\hline Multi-Allelic Variants (MAVs) & 616 & 526 & $3 *$ \\
\hline Insertions & 1,110 & 1,044 & 36 \\
\hline
\end{tabular}




\begin{tabular}{|l|r|r|r|} 
Deletions & 2,799 & 2,677 & 95 \\
\hline Total & 661,126 & 610,771 & 594,361 \\
\hline
\end{tabular}

\section{GSA assays for transitions perform better than do those for transversions}

352 Classifying the GSA-detected SNVs as transitions (purine-to-purine OR pyrimidine-to-

353 pyrimidine) or transversions (purine-to-pyrimidine or vice versa) identified 522,938 (79.6\%)

354 assays for transitions and 133,663 (20.4\%) for transversions. 476,908 (91.2\%) transition assays

355 and 117,322 (87.8\%) transversion assays passed cluster file QC and had genotypes present in the

$3561 \mathrm{KG}$.

357

358 Assays for transitions performed better than those for transversions across all performance

359 metrics. Overall concordance, sensitivity, specificity and positive predictive value for transitions

360 versus transversions were 0.9985 vs. $0.9965,0.9982$ vs. $0.9965,0.9994$ vs. 0.9985 and 0.998 vs.

361 0.996, respectively (Figure 4B). The assays for transversions between complementary

362 nucleotides (i.e., A>T, T>A, C>G, G>C; see Supplementary Materials - Section 7) had lower

363 sensitivity $(<0.99)$ and lower cluster file QC pass rate $(66-73 \%$; Table 6) than did those for other

364 transversions.

366 Table 6. Distribution of GSA (reference (Ref) to alternate (Alt) allele) SNV assays present in the 367 $1 \mathrm{KG}$ Phase 3 data versus number of assays passing QC

\begin{tabular}{|l|c|c|c|c|c|}
\hline & ReflAlt & Purine & Purine & Pyrimidine & Pyrimidine \\
\hline Purine & A & - & G & C & T \\
\hline Purine & G & $\begin{array}{c}101,994 / 111,493 \\
(91 \%)\end{array}$ & $\begin{array}{c}25,107 / 28,214 \\
(89 \%)\end{array}$ & $\begin{array}{c}1,368 / 2,065 \\
(66 \%)\end{array}$ \\
\hline Pyrimidine & C & $\begin{array}{c}30,130 / 33) \\
(90 \%)\end{array}$ & - & $\begin{array}{c}2,404 / 3,280 \\
(73 \%)\end{array}$ & $\begin{array}{c}29,629 / 33,115 \\
(89 \%)\end{array}$ \\
\hline
\end{tabular}




\begin{tabular}{|c|c|c|c|c|c|}
\hline Pyrimidine & $\mathbf{T}$ & $\begin{array}{c}1,362 / 2,074 \\
(66 \%)\end{array}$ & $\begin{array}{c}24,983 / 28,133 \\
(89 \%)\end{array}$ & $\begin{array}{c}102,172 / 112,009 \\
(91 \%)\end{array}$ & - \\
\hline
\end{tabular}

371 Using the allele frequency in the $1 \mathrm{KG}$ as a surrogate for the general population variant allele

372 frequency, we interrogated the effect of alternate allele (variant allele) frequency on the

373 performance metrics. 643,012 GSA SNV assays were binned according to the alternate allele

374 frequency extracted from the $1 \mathrm{KG} \mathrm{VCF}$ file (allele frequency * 100): (a) [0-0.1\%], (b) (0.1-

$3751 \%]$, (c) (1-5\%], (d) (5-10\%], (e) (10-20\%], (f) (20-30\%], (g) (30-40\%], (h) (40-50\%], (i) (50-

376 60\%], (j) (60-70\%], (k) (70-80\%], (l) (80-90\%], and (m) (90-100\%] (Table 7). On average the

377 QC process removed 7-8\% of assays from each bin. The bins [0-0.1\%] and (90-100\%] had 2\%

378 and $12 \%$ respectively removed (Table 7); this might reflect the small number of assays in these

379 bins (17,830 and 4,552, respectively). Consistent with previous publications (Ritchie et al. 2011),

380 the average performance metrics for GSA assays passing cluster file QC in each bin showed that

381 PPV and sensitivity suffered when the alternate allele frequency was $<5 \%$, whereas specificity

382 and concordance declined as the alternate allele frequency increased (Figure 4C).

Table 7. Number of GSA assays and their relative percentages binned by alternate allele frequency in $1 \mathrm{KG}$ Phase 3 data.

\begin{tabular}{|l|r|r|r|}
\hline $\begin{array}{c}\text { Alternate allele } \\
\text { frequency bins (\%) }\end{array}$ & $\begin{array}{c}\text { All GSA } \\
\text { assays and in } \\
\text { 1KG }\end{array}$ & $\begin{array}{c}\text { GSA pass } \\
\text { QC and in } \\
\mathbf{1 K G}\end{array}$ & $\begin{array}{c}\text { Percent } \\
\text { assays that } \\
\text { failed QC }\end{array}$ \\
\hline $0-0.1$ & 17,830 & 17,454 & $2 \%$ \\
\hline $0.1-1$ & 148,959 & 138,342 & $7 \%$ \\
\hline $1-5$ & 113,374 & 104,272 & $8 \%$ \\
\hline $5-10$ & 63,688 & 58,421 & $8 \%$ \\
\hline $10-20$ & 84,729 & 78,631 & $7 \%$ \\
\hline
\end{tabular}




\begin{tabular}{|l|r|r|r|}
$20-30$ & 56,601 & 52,398 & $7 \%$ \\
\hline $30-40$ & 39,684 & 36,620 & $8 \%$ \\
\hline $40-50$ & 30,095 & 27,476 & $9 \%$ \\
\hline $50-60$ & 25,078 & 23,053 & $8 \%$ \\
\hline $60-70$ & 21,944 & 20,134 & $8 \%$ \\
\hline $70-80$ & 20,866 & 19,210 & $8 \%$ \\
\hline $80-90$ & 15,612 & 14,312 & $8 \%$ \\
\hline $90-100$ & 4,552 & 4,023 & $12 \%$ \\
\hline Total & $\mathbf{6 4 3 , 0 1 2}$ & $\mathbf{5 9 4 , 3 4 6}$ & $\mathbf{8 \%}$ \\
\hline
\end{tabular}

388 Among the 594,346 GSA SNV assays with IKG genotypes, 476,707 had a PPV equal to 1 (zero 389 false positives) based on concordance with the 1KG and rWGS genotypes (Supplementary Table 390 S8). We observed that $81 \%$ of GSA assays in the [0 - 0.1\%] and $28 \%$ of GSA assays in the $391(0.1 \%-1 \%]$ bins had PPV $<1$ (Figure 5 and Table 9), whereas other allele frequency bins had an 392 average of $13 \%$ (range: 8-17\%) with a PPV<1 (Table 8). These results are consistent with prior 393 observations showing that accurate calling of rare alleles (MAF < 0.01 ) by genotyping arrays is 394 compromised by low genotype frequencies and an absence of the homozygous alternate alleles 395 needed for construction of cluster files $(22,39)$.

397 Table 8. GSA assays with a $P P V=1$ based on concordance with the $1 \mathrm{KG}$ Phase 3 data and the 398 rWGS data. Data is binned by alternate allele frequency.

\begin{tabular}{|l|r|r|r|c|}
\hline Allele frequency bins & $\begin{array}{c}\text { Total QC pass } \\
\text { assays }\end{array}$ & $\begin{array}{c}\text { Assays with } \\
\text { PPV =1; } \\
\text { GSA vs. } \\
\text { 1KG and } \\
\text { GSA vs. } \\
\text { rWGS }\end{array}$ & \% left & \% filtered \\
\hline $0-0.1$ & 17,454 & 3,283 & 19 & 81 \\
\hline $0.1-1$ & 138,342 & 99,508 & 72 & 28 \\
\hline $1-5$ & 104,272 & 88,235 & 85 & 15 \\
\hline $5-10$ & 58,421 & 48,532 & 83 & 17 \\
\hline $10-20$ & 78,631 & 66,027 & 84 & 16 \\
\hline $20-30$ & 52,398 & 44,511 & 85 & 15 \\
\hline
\end{tabular}




\begin{tabular}{|l|r|r|r|c|}
$30-40$ & 36,620 & 31,223 & 85 & 15 \\
\hline $40-50$ & 27,476 & 23,563 & 86 & 14 \\
\hline $50-60$ & 23,053 & 20,041 & 87 & 13 \\
\hline $60-70$ & 20,134 & 17,799 & 88 & 12 \\
\hline $70-80$ & 19,210 & 17,318 & 90 & 10 \\
\hline $80-90$ & 14,312 & 13,103 & 92 & 8 \\
\hline $90-100$ & 4,023 & 3,564 & 89 & 11 \\
\hline Total & 594,346 & 476,707 & & \\
\hline
\end{tabular}

401 Table 9. Summary of performance metrics for GSA and rWGS relative to $1 \mathrm{KG}$ Phase 3 data

\begin{tabular}{|l|c|c|}
\hline Performance metrics & $\begin{array}{c}\text { Global Screening Array } \\
\text { (GSA) vs. 1KG } \\
\text { Mean }( \pm \text { std.dev) }\end{array}$ & $\begin{array}{c}\text { Whole Genome } \\
\text { Sequencing (WGS) } \\
\text { vs. 1KG } \\
\text { Mean }( \pm \text { std.dev) }\end{array}$ \\
\hline Concordance & $0.9932( \pm 0.0005)$ & $0.9981( \pm 0.0005)$ \\
\hline Sensitivity & $0.9927( \pm 0.0007)$ & $0.9981( \pm 0.0005)$ \\
\hline Specificity & $0.9957( \pm 0.0003)$ & $0.9991( \pm 0.0003)$ \\
\hline $\begin{array}{l}\text { Positive Predictive } \\
\text { Value (PPV) }\end{array}$ & $0.9892( \pm 0.0008)$ & $0.9977( \pm 0.0007)$ \\
\hline
\end{tabular}

403 GSA assays interrogating low-complexity genomic regions perform poorer than other

404 assays

405 To determine assay performance characteristics within repetitive regions of the genome, we

406 intersected GSA assays with annotated low complexity regions (LCRs) including simple repeats,

407 microsatellites, and repeat masked (RepeatMasker-defined) regions in the human genome. Of a

408 total of 594,346 assays passing QC and present in the $1 \mathrm{KG}, 203,901(\sim 34 \%)$ assessed a variant

409 within one of the three annotation classes. 201,579 GSA assays mapped within the

410 RepeatMasker class. Overlapping partially with the other two classes, 431 GSA assays mapped

411 within the simple repeat class. GSA assays targeting genotypes within each LCR class had 
412 poorer performance metrics than did assays interrogating genotypes outside of these regions

413 (Figure 4D).

414

415 rWGS performed better than GSA relative to the benchmark dataset 1KG

416 rWGS data corresponding to GSA assays passing QC were extracted from the rWGS gVCF files

417 and compared to the $1 \mathrm{KG}$. Restricting the analyses to GSA assays for which >90\% of rWGS

418 samples had genotype data defined 602,582 assays and excluded 38,093 GSA assays. An

419 additional 9,642 assays on the chromosome $\mathrm{X}$ were excluded due to discrepancies in genotype

420 representation in comparison datasets. For the remaining 592,940 autosomal assays, the rWGS

421 genotypes with $>20 x$ coverage and a Phred score $>30$ were used for calculation of performance

422 metrics. These analyses, i.e., GSA vs. 1KG and rWGS vs. 1KG, showed consistent average

423 metrics and small standard deviations among datasets (Table 9).

424

425 For the 256 Coriell samples with $1 \mathrm{KG}$ data, we observed that rWGS performed better than GSA

426 across all 4 performance metrics (Figure 6A and Table 9). Overall average concordance,

427 sensitivity, and specificity for rWGS vs. 1KG were 0.9981, 0.9981 and 0.9991, respectively,

428 whereas for GSA vs. $1 \mathrm{KG}$, they were 0.9932, 0.9927, and 0.9957, respectively. PPV was 0.9977

429 for rWGS vs. $1 \mathrm{KG}$ and was 0.9892 for GSA vs. 1KG (Table 9).

430

431 Over $82 \%$ of all GSA assays have a PPV = 1

432 We compared the GSA and rWGS genotypes to the $1 \mathrm{KG}$ and computed the PPV. As shown in

433 Figure 6B, over $82 \%(476,828)$ of assays had a PPV of 1 for both the GSA and rWGS. 
434 Approximately $1.5 \%(8,710)$ of rWGS assays had a PPV of 1 when GSA was 0 , whereas only

$4350.12 \%(699)$ of GSA assays did when rWGS was 0.

437 GSA MAP59 secondary findings validated using rWGS, pWGS, and 1KG

438 Given that $>80 \%$ of GSA assays have a PPV=1, we assessed rare variation detection within the

43959 medically actionable predisposition genes (MAP59) defined by the American College of

440 Medical Genetics (ACMG) (37). Given the expected secondary finding rate of 1\%-2\% (40-42)

441 and the limited genomic space profiled by the GSA, we hypothesized $2-3$ or fewer samples

442 with GSA-detectable variants in the 261 cohort. Additionally, we hypothesized that comparison

443 of these data to the $1 \mathrm{KG}$ and the rWGS data identifies false negative and false positive variants

444 as well as pathogenic variation undetected by the GSA. Focusing on nucleotides with $>20 x$

445 rWGS coverage (Figure 7), we found that an average of 6,347 ( \pm 88 ) sites were genotyped by

446 both rWGS and GSA in any given DNA sample. The GSA vs. rWGS average concordance,

447 sensitivity, specificity, and PPV were 0.99897, 0.99367, 0.99962, and 0.9946, respectively.

449 For clinically reportable rare variants curated into the managed variant list (MVL), the GSA and

450 rWGS were concordant for a heterozygous variant (MUTYH p.(Gly368Asp); rs36053993) in

451 three samples and across GSA replicates. Two of the 3 samples had $1 \mathrm{KG}$ data and were

452 concordant; one of these two had pWGS data that was also concordant. Highlighting the

453 potential for false positives, rWGS and $1 \mathrm{KG}$ data refuted a GSA call of $P K P 2$ p.(Arg355Ter)

454 (rs754912778) in one sample. Conversely, highlighting the potential for false negatives, rWGS

455 and $1 \mathrm{KG}$ detected two variants that were not detected by GSA: RB1 p.(Arg661Trp)

456 (rs137853294), which the GSA called homozygous reference in triplicate, and MUTYH 
457 p.(Pro391Leu) (rs529008617), which the GSA called "no-call" in triplicate. In summary, the 458 GSA identified 1 pathogenic variant (true positive), 1 false positive, and 2 false negatives (2 459 assayed and missed) among the MAP59.

460

461 To identify rare pathogenic variation discovered by rWGS and not assayed by the GSA (lack of

462 probe coverage), we intersected rWGS data with ClinVar pathogenic variation and found 4

463 heterozygote variants not assayed by the GSA. These were APOB p.(Arg3527Trp)

464 (rs144467873), SDHAF2 p.(Asn103GlufsTer4) (frameshift insertion; rs753554501), BRCA2

465 p.(Ser1748Ter) (insertion (NM_000059.3:c.5241_5242insTA); rs749980674) and ATP7B

466 p.(Thr991Met) (rs41292782). One of these 4 (APOB p.(Arg3527Trp); rs144467873) was present

467 in $1 \mathrm{KG}$. The $A T P 7 B$ p.(Thr991Met) (rs41292782) variant was likely absent from the $1 \mathrm{KG}$ due to

468 poor coverage. In summary, rWGS identified 7 rare pathogenic variants in MAP59 genes in 9

469 samples; the GSA lacked assays for 4 rare pathogenic variants detected by WGS.

470

471 The rWGS rate of detection of rare pathogenic variants in the MAP59 genes was $0.034(3.4 \%) ; 7$

472 variants in 9 samples from a population of 261. Removing the 3 variants that were not

473 independently confirmed by the $1 \mathrm{KG}$ due to lack of $1 \mathrm{KG}$ data gives 4 pathogenic variants in 5

474 individuals from a population of 261 or a rate of $0.019(1.9 \%)$. This range $(0.019-0.034)$ of

475 pathogenic variants in the MAP59 genes is consistent with the published discovery rate $(40,41$, $47643,44)$.

477

478

479 


\section{Discussion}

481 We report an approach to analytical validation of the GSA through quality analyses and through

482 assessment of performance by comparison to benchmark datasets and independent whole-

483 genome sequencing data. To the best of our knowledge, this is the first comprehensive analytical

484 validation of the GSA for clinical genotyping. Our findings support and extend recently reported

485 research studies assessing the utility of the GSA for genetic screening in primary

486 immunodeficiency (45), for population-based genomic screening for rare and medically relevant

487 variation (46), and for detecting rare and clinically relevant markers in multiethnic Indian

488 populations (47).

489

490 In our study we used call rate and sample contamination as preliminary parameters of quality

491 control for genotype analysis. Call rate is a primary quality control parameter in all genotyping

492 studies $(12,13)$. A high threshold for call rate not only ensures inclusion of samples with high

493 quality genotype data but also allows, independent of sample DNA quality, for detection of

494 assays that perform poorly. Additionally, sample contamination detection (14) is key in

495 preventing return of false positive genotypes and is demonstrated by our results. While more

496 advanced quality control methods such as Hardy-Weinberg Equilibrium (HWE) test (15),

497 likelihood of error (19), departure from Mendelian inheritance and pedigree information are used

498 in various research studies $(4,20)$, they are implemented in analyses that follow genotype

499 generation and are dependent on what analyses are subsequently performed using the genotype

500 data. HWE is used to detect genotypes that deviate from the expectation of HWE, and it is

501 typically applied to variants with a MAF of greater than 0.05 (12). Consequently, because of our

502 interest in variants of lower MAF, we did not implement this QC metric; however, HWE might 
503 be useful within certain cut-offs for MAF as implemented by Suratannon et. al. (45) and Narang

504 et. al. (47). Similarly, Mendelian inheritance and pedigree information quality control are critical

505 for linkage and segregation analyses and did not apply to our individual-focused assay.

506

507 This evaluation of GSA data is consistent with previous studies that demonstrated utility of

508 sample data quality metrics like genotype call-rate, p10GC, and DNA contamination detection

$509(11,22)$. By analysis of replicates, we show that the majority of the GSA data are highly

510 reproducible. Outliers arose either from positioning along the edges of the Illumina BeadChip or

511 from contamination. Characterization of each GSA assay by variation class, type, genomic DNA

512 complexity, and alternate allele frequency showed that the GSA has the highest performance for

513 SNVs and transition nucleotide changes in genomic regions of high complexity. In contrast,

514 assays interrogating low-complexity regions, rare alleles, or transversions performed poorly.

515 Transversions between complementary nucleotides likely performed poorly because of the

516 characteristics of the assays for these particular transversions (Supplementary Materials -

517 Section 7). Also, consistent with previous reports $(48-50)$, assays for rare alleles $(<0.001)$ had

518 lower performance and might be improved by using algorithms for rare variant detection (10, 31,

51932 ) or joint-calling (22) rather than the default genotype caller (GenCall). These should be

520 considered in the future to improve detection of rare variants by genotyping chips.

521

522 The analytical framework implemented in this study followed a three-way analysis (GSA-rWGS-

$5231 \mathrm{KG}$ ) to assess the strengths and limitations of individual GSA assays. Unlike many published

524 analyses in which WGS is the test dataset and the BeadArray genotypes are the truth $(25-27,30)$,

525 our study had the BeadArray as the test dataset and WGS as the truth. The reversal of test and 
526 truth datasets is a major challenge for comparing our results to the published literature. To

527 overcome this challenge, we ensured that the rWGS data had performance metrics

528 (concordance $=0.9981)$ comparable to that previously published $($ concordance $=0.9984(25))$. The

529 three-way analysis framework also allowed detection of false positive and false negative

530 genotypes on the GSA platform. Though not evaluated in the current study, the three-way

531 comparison framework in our analysis allows for modeling of genotyping-error specific to

532 variation classes and categories triaged during characterization of the GSA.

533

534 Over $82 \%$ of assays on the GSA returned genotypes with a high positive predictive value (PPV).

535 The GSA detected some pathogenic variation (MAP59) in the test dataset of 261 Coriell

536 samples, and these variants were independently validated by either the 1KG data or the rWGS /

537 pWGS data or both. Although we attempted to compare GSA results to other chip results

538 (example HumanExome chip), the comparison to previous work was impeded by differences in

539 probe content and density as well as chip design (e.g., 610k assays on GSA, vs. 247k assays on

540 HumanExome chip). Some of the pros and cons of using the GSA are summarized in Table 10

541 below.

542

543 Table 10. Pros and cons of arrays vs. whole genome sequencing (51)

\begin{tabular}{|c|c|c|}
\hline Feature & SNP arrays (GSA) & WGS \\
\hline Cost & Lower cost & Higher cost \\
\hline $\begin{array}{l}\text { Genomic } \\
\text { coverage }\end{array}$ & $\begin{array}{l}\text { Best for variants for which } \\
\text { DNAs of all genotype } \\
\text { combinations are available, i.e., } \\
\text { not robust for rare variants } \\
\text { Requires prior knowledge of the } \\
\text { variant, i.e., unable to detect } \\
\text { private variants not previously } \\
\text { reported } \\
\text { Reduced accuracy in genomic } \\
\text { regions of low complexity }\end{array}$ & $\begin{array}{l}\text { Appropriate for detection of nearly all } \\
\text { genetic variation in the genome depending } \\
\text { on the depth of sequencing, i.e., not robust } \\
\text { for difficult to sequence regions. } \\
\text { - Reduced accuracy in genomic regions of } \\
\text { low complexity }\end{array}$ \\
\hline
\end{tabular}




\begin{tabular}{|l|l|ll|}
\hline Analyses & $\begin{array}{l}\text { Well established analytical protocols and } \\
\text { tools for data analyses }\end{array}$ & $\begin{array}{l}\text { High computational costs and greater } \\
\text { analytical complexity }\end{array}$ \\
& $\begin{array}{l}\text { Larger multiple testing burden when } \\
\text { conducting single-variant tests }\end{array}$ \\
& $\begin{array}{l}\text { Greater costs to store, process, analyze and } \\
\text { interpret the resulting data }\end{array}$ \\
\hline Suitability & $\begin{array}{l}\text { Screening } \\
\text { Analyzing known or candidate } \\
\text { associations in large cohorts } \\
\text { Detecting low-frequency, } \\
\text { common variant associations in } \\
\text { large sample sizes }\end{array}$ & $\begin{array}{l}\text { Diagnostic testing } \\
\text { Detecting and fine-mapping rare variants }\end{array}$ \\
\hline
\end{tabular}

545 The test characteristics of the GSA compared to WGS clearly show that the GSA is not a

546 diagnostic genomic test for individuals with rare disorders because, as shown by our MAP59

547 results and recent research studies $(45,46)$, it lacks robustness for genotyping rare variants as

548 well as probes for detection of private familial disease variants. On the other hand, we show that

549 the GSA has the analytical robustness to serve as a clinical screen for genotypes for which one

550 can establish robust cluster files for the AA, AB, and BB genotypes. This is most easily

551 accomplished for more common genotypes that contribute to polygenic predispositions to

552 disease, particularly common diseases. Screening of an asymptomatic population to assess the

553 likelihood of predisposition to a disease is well established within medicine, and examples

554 include newborn screening for inborn errors of metabolism, mammography for breast cancer,

555 and cholesterol levels for coronary artery disease (52, 53). A major objective of screening tests is

556 to reduce morbidity and mortality in the subject population through risk stratification to target

557 surveillance, early detection, and treatment. With the characterization of genomic risk for drug

558 responsiveness and predisposition to various cancers and cardiovascular disease (54-56), we

559 propose that the GSA offers a potential clinical tool for genomic screening. 


\section{Limitations of our study}

564 Our comparison of BeadChip arrays to NGS and benchmark datasets has some limitations.

565 Firstly, we evaluated our dataset using accepted algorithms. This did not take into account the

566 benefits of consensus genotyping by multiple algorithms for GSA or NGS data; Hwang et al.

567 found that consensus genotyping minimized false findings (49). Secondly, cell-line derived

568 variation or low-level somatic variation might also have contributed to differences between

569 datasets (25). Thirdly, we did not analyze variants close to or overlapping other variation in the

570 same location, e.g., insertions/deletions and copy number variation, because these loci are

571 eukaryotic mutation hotspots (57). Fourthly, our analysis would benefit from comparison to

572 variant benchmark datasets defined in more recent publications (49) and to NIST / GiAB

573 datasets.

574

575

\section{Conclusions}

576 We established the analytical validity of the GSA via a systematic approach utilizing benchmark

577 and rWGS data to evaluate the performance of each assay. We highlight that although the GSA

578 assays within particular genotype classes, particularly those interrogating rare variants,

579 transversions, and variants within low-complexity regions, need careful evaluation GSA assays

580 can be analytically validated to clinically screen for common genotypes predisposing to disease.

\section{List of abbreviations}

584 SNV - Single Nucleotide Variation 
585 MAV - Multi-allelic variant

586 DEL - Deletion

587 INS - Insertion

588 PPV - Positive predictive value

589 QC - Quality control

590 NGS - Next-generation sequencing

591 GSA - Global screening array

592 rWGS - Whole-genome sequencing

$593 \quad \mathrm{IKG}-1000$ Genomes

594 HTS - High-throughput sequencing

595 VCF - Variant call file

596 AWS - Amazon web services

597 PCA - Principal component analysis

598 TP - True positive

$599 \mathrm{TN}-$ True negative

600 FP - False positive

$601 \quad F N-$ False negative

602 MAP - Medically actionable predisposition

603 LCR - Low-complexity regions

$604 \mathrm{GiAB}-$ Genome in a bottle 


\section{Ethics approval}

607 On June 6, 2018, the Sanford Health IRB determined that the proposed activity,

608 "STUDY00001343: Framework for Analytical Validation of SNP Arrays" was not human

609 research and therefore Sanford Health IRB review and approval was not required.

\section{Consent to participate}

612 On June 6, 2018, the Sanford Health IRB determined that the proposed activity,

613 "STUDY00001343: Framework for Analytical Validation of SNP Arrays" was not human

614 research and therefore Sanford Health IRB review and approval was not required. For studies

615 deemed not human research, consent is deemed unnecessary under the 2018 Common Rule, (45

616 CFR 46).

\section{Consent to publish}

619 Not applicable.

620

\section{Availability of data and materials}

622 The datasets generated and analyzed during the current study are not publicly available due to

623 enormous size of datasets (whole genome sequence and genotyping chip array data: over 22

624 terabytes (TB)) but are available from the corresponding author on reasonable request. All

625 datasets generated and analyzed during the current study are archived privately on Amazon Web

626 Services Storage 3 (AWS S3). Data supporting our findings when size was not a limitation were

627 made available in Supplementary Material. 


\section{Competing interests}

630 The authors declare that they have no competing interests.

\section{Funding}

633 Sanford Health funded the design of the study and collection, analysis, and interpretation of data 634 and in writing the manuscript.

\section{Authors' contributions}

637 CFB, CH, LC, and PFC conceived the hypothesis tested in this study. PFC and LC developed the

638 methodology to test that hypothesis. MMS, LMF, KPL, JML generated the data and performed 639 the preliminary data analysis. REP coordinated initial data validation and GSA manifest file 640 curation. PFC, DEC, SB, KM, SG, FGF and MMS aided in data analysis. PFC wrote the 641 manuscript with input and revisions provided by CFB, KPL, JML, DEC, KM, MMS, FGF and

642 LC. LC, $\mathrm{CH}$ and CFB aided in the relevance of conclusions drawn from data analysis. All 643 authors read and approved the final manuscript.

\section{Acknowledgements}

646 We acknowledge Drs. Huilin Chin (Khoo Teck Puat-National University Children's Medical

647 Institute, National University Hospital, Singapore), Sylvie Langlois (Provincial Medical Genetics

648 Program, BC Women's Hospital, University of British Columbia, Canada) and Blake Atwood

649 (Imagenetics, Sanford Health) for critique of the manuscript. Additionally, we acknowledge

650 valuable help and support from the following colleagues and collaborators: Suruchi Ahuja, 
651 Christina Carlson, Chun H Chan, Megan Cornwell, Chris Deschler (GenomeNext), James

652 Hirmas (GenomeNext), Ryan Kelly (Illumina), Danny W Lee, Dmitry Lyalin, Michael Mboob,

653 Lexie Mohror, Michele M Moore, Lisa Mullineaux, Jeremy Pierce (Illumina), Jennifer Reiner,

654 Murat Sincan, Sherin Shabaan, and Bethany Tucker.

\section{References}

658 1. Muyas F, Bosio M, Puig A, Susak H, Domènech L, Escaramis G, et al. Allele balance 659 bias identifies systematic genotyping errors and false disease associations. Hum Mutat.

660 2019;40(1):115-26.

661 2. Yan Q, Chen R, Sutcliffe JS, Cook EH, Weeks DE, Li B, et al. The impact of genotype

662 calling errors on family-based studies. Sci Rep. 2016;6:28323.

663 3. Walters K. The effect of genotyping error in sib-pair genomewide linkage scans depends 664 crucially upon the method of analysis. J Hum Genet. 2005;50(7):329-37.

665 4. Saunders IW, Brohede J, Hannan GN. Estimating genotyping error rates from Mendelian errors in SNP array genotypes and their impact on inference. Genomics. 2007;90(3):291-6. 5. Pompanon F, Bonin A, Bellemain E, Taberlet P. Genotyping errors: causes, consequences and solutions. Nat Rev Genet. 2005;6(11):847-59.

6. Mayer-Jochimsen M, Fast S, Tintle NL. Assessing the impact of differential genotyping errors on rare variant tests of association. PLoS One. 2013;8(3):e56626.

7. Hong H, Su Z, Ge W, Shi L, Perkins R, Fang H, et al. Assessing batch effects of genotype calling algorithm BRLMM for the Affymetrix GeneChip Human Mapping $500 \mathrm{~K}$ array set using 270 HapMap samples. BMC Bioinformatics. 2008;9 Suppl 9:S17.

8. Fadista J, Bendixen C. Genomic Position Mapping Discrepancies of Commercial SNP Chips. PLoS One. 2012;7(2).

9. Chan AW, Williams AL, Jannink J-L. A statistical framework for detecting mislabeled and contaminated samples using shallow-depth sequence data. BMC Bioinformatics.

679 10. Ritchie ME, Liu R, Carvalho BS, Australia, New Zealand Multiple Sclerosis Genetics C, 680 Irizarry RA. Comparing genotyping algorithms for Illumina's Infinium whole-genome SNP 681 BeadChips. BMC Bioinformatics. 2011;12:68.

682 11. Igo RP, Cooke Bailey JN, Romm J, Haines JL, Wiggs JL. Quality Control for the 683 Illumina HumanExome BeadChip. Curr Protoc Hum Genet. 2016;90:2.14.1-2..6.

684 12. Guo Y, He J, Zhao S, Wu H, Zhong X, Sheng Q, et al. Illumina human exome genotyping array clustering and quality control. Nat Protoc. 2014;9(11):2643-62.

13. Gudiseva HV, Hansen M, Gutierrez L, Collins DW, He J, Verkuil LD, et al. Saliva DNA 688 quality and genotyping efficiency in a predominantly elderly population. BMC Med Genomics. 2016;9:17. 
14. Jun G, Flickinger M, Hetrick KN, Romm JM, Doheny KF, Abecasis GR, et al. Detecting and estimating contamination of human DNA samples in sequencing and array-based genotype data. Am J Hum Genet. 2012;91(5):839-48.

692 15. Chen B, Cole JW, Grond-Ginsbach C. Departure from Hardy Weinberg Equilibrium and Genotyping Error. Front Genet. 2017;8.

694 16. Wang J, Shete S. Testing Departure from Hardy-Weinberg Proportions. Methods Mol 695 Biol. 2017;1666:83-115.

696 17. Zhao S, Jing W, Samuels DC, Sheng Q, Shyr Y, Guo Y. Strategies for processing and quality control of Illumina genotyping arrays. Brief Bioinform. 2018;19(5):765-75.

18. Sellick GS, Goldin LR, Wild RW, Slager SL, Ressenti L, Strom SS, et al. A high-density SNP genome-wide linkage search of 206 families identifies susceptibility loci for chronic lymphocytic leukemia. Blood. 2007;110(9):3326.

19. Ehm MG, Kimmel M, Cottingham RW. Error detection for genetic data, using likelihood methods. American Journal of Human Genetics. 1996;58(1):225-34.

20. Hao K, Li C, Rosenow C, Hung Wong W. Estimation of genotype error rate using samples with pedigree information--an application on the GeneChip Mapping 10K array. Genomics. 2004;84(4):623-30.

21. Laurie CC, Doheny KF, Mirel DB, Pugh EW, Bierut LJ, Bhangale T, et al. Quality control and quality assurance in genotypic data for genome-wide association studies. Genetic Epidemiology. 2010;34(6):591-602.

22. Grove ML, Yu B, Cochran BJ, Haritunians T, Bis JC, Taylor KD, et al. Best practices and joint calling of the HumanExome BeadChip: the CHARGE Consortium. PLoS One. 2013;8(7):e68095.

23. O'Rawe J, Jiang T, Sun G, Wu Y, Wang W, Hu J, et al. Low concordance of multiple variant-calling pipelines: practical implications for exome and genome sequencing. Genome Med. 2013;5(3):28.

24. Pongpanich M, Sullivan PF, Tzeng J-Y. A quality control algorithm for filtering SNPs in genome-wide association studies. Bioinformatics. 2010;26(14):1731-7.

25. Eberle MA, Fritzilas E, Krusche P, Källberg M, Moore BL, Bekritsky MA, et al. A reference data set of 5.4 million phased human variants validated by genetic inheritance from sequencing a three-generation 17-member pedigree. Genome Res. 2017;27(1):157-64.

26. Zook JM, Chapman B, Wang J, Mittelman D, Hofmann O, Hide W, et al. Integrating human sequence data sets provides a resource of benchmark SNP and indel genotype calls. Nature Biotechnology. 2014;32(3):246-51.

27. Krusche P, Trigg L, Boutros PC, Mason CE, De La Vega FM, Moore BL, et al. Best practices for benchmarking germline small-variant calls in human genomes. Nature Biotechnology. 2019;37(5):555-60.

28. Stephens ZD, Hudson ME, Mainzer LS, Taschuk M, Weber MR, Iyer RK. Simulating Next-Generation Sequencing Datasets from Empirical Mutation and Sequencing Models. PLoS One. 2016;11(11):e0167047.

29. Highnam G, Wang JJ, Kusler D, Zook J, Vijayan V, Leibovich N, et al. An analytical framework for optimizing variant discovery from personal genomes. Nat Commun. 2015;6:6275. 30. Zook JM, McDaniel J, Olson ND, Wagner J, Parikh H, Heaton H, et al. An open resource for accurately benchmarking small variant and reference calls. Nature Biotechnology. 2019;37(5):561-6. 
31. Zhou J, Tantoso E, Wong L-P, Ong RT-H, Bei J-X, Li Y, et al. iCall: a genotype-calling algorithm for rare, low-frequency and common variants on the Illumina exome array. Bioinformatics. 2014;30(12):1714-20. 32. Goldstein JI, Crenshaw A, Carey J, Grant GB, Maguire J, Fromer M, et al. zCall: a rare variant caller for array-based genotypingGenetics and population analysis. Bioinformatics. 2012;28(19):2543-5.

33. Mao Q, Ciotlos S, Zhang RY, Ball MP, Chin R, Carnevali P, et al. The whole genome sequences and experimentally phased haplotypes of over 100 personal genomes. Gigascience. 2016;5(1):42.

34. Kelly BJ, Fitch JR, Hu Y, Corsmeier DJ, Zhong H, Wetzel AN, et al. Churchill: an ultrafast, deterministic, highly scalable and balanced parallelization strategy for the discovery of human genetic variation in clinical and population-scale genomics. Genome Biol. 2015;16:6. 35. Reich D, Price AL, Patterson N. Principal component analysis of genetic data. Nature Genetics. 2008;40(5):491-2.

36. Nyamundanda G, Poudel P, Patil Y, Sadanandam A. A Novel Statistical Method to Diagnose, Quantify and Correct Batch Effects in Genomic Studies. Sci Rep. 2017;7(1):10849.

37. Kalia SS, Adelman K, Bale SJ, Chung WK, Eng C, Evans JP, et al. Recommendations for reporting of secondary findings in clinical exome and genome sequencing, 2016 update (ACMG SF v2.0): a policy statement of the American College of Medical Genetics and Genomics. Genet Med. 2017;19(2):249-55.

38. Church GM. The personal genome project. Mol Syst Biol. 2005;1:2005.0030.

39. Perreault L-PL, Legault M-A, Barhdadi A, Provost S, Normand V, Tardif J-C, et al. Comparison of genotype clustering tools with rare variants. BMC Bioinformatics. 2014;15:52. 40. Amendola LM, Dorschner MO, Robertson PD, Salama JS, Hart R, Shirts BH, et al. Actionable exomic incidental findings in 6503 participants: challenges of variant classification. Genome Res. 2015;25(3):305-15.

41. Hart MR, Biesecker BB, Blout CL, Christensen KD, Amendola LM, Bergstrom KL, et al. Secondary findings from clinical genomic sequencing: prevalence, patient perspectives, family history assessment, and health-care costs from a multisite study. Genet Med. 2019;21(5):110010 .

42. Sapp JC, Johnston JJ, Driscoll K, Heidlebaugh AR, Miren Sagardia A, Dogbe DN, et al. Evaluation of Recipients of Positive and Negative Secondary Findings Evaluations in a Hybrid CLIA-Research Sequencing Pilot. Am J Hum Genet. 2018;103(3):358-66.

43. Dorschner MO, Amendola LM, Turner EH, Robertson PD, Shirts BH, Gallego CJ, et al. Actionable, pathogenic incidental findings in 1,000 participants' exomes. American Journal of Human Genetics. 2013;93(4):631-40.

44. Kim J, Luo W, Wang M, Wegman-Ostrosky T, Frone MN, Johnston JJ, et al. Prevalence of pathogenic/likely pathogenic variants in the 24 cancer genes of the ACMG Secondary Findings v2.0 list in a large cancer cohort and ethnicity-matched controls. Genome Med. 2018;10(1):99.

45. Suratannon N, van Wijck RTA, Broer L, Xue L, van Meurs JBJ, Barendregt BH, et al. Rapid Low-Cost Microarray-Based Genotyping for Genetic Screening in Primary Immunodeficiency. Front Immunol. 2020;11:614.

46. Bowling KM, Thompson ML, Gray DE, Lawlor JMJ, Williams K, East KM, et al. Identifying rare, medically relevant variation via population-based genomic screening in Alabama: opportunities and pitfalls. Genet Med. 2020. 
781 spectrum of rare and clinically relevant markers in multiethnic Indian populations (ClinIndb): A 782 resource for genomic medicine in India. Hum Mutat. 2020;41(11):1833-47.

783 48. DePristo MA, Banks E, Poplin R, Garimella KV, Maguire JR, Hartl C, et al. A

784 framework for variation discovery and genotyping using next-generation DNA sequencing data.

785 Nature Genetics. 2011;43(5):491-8.

786 49. Hwang K-B, Lee I-H, Li H, Won D-G, Hernandez-Ferrer C, Negron JA, et al.

787 Comparative analysis of whole-genome sequencing pipelines to minimize false negative 788 findings. Sci Rep. 2019;9(1):3219.

78950 . Korn JM, Kuruvilla FG, McCarroll SA, Wysoker A, Nemesh J, Cawley S, et al.

790 Integrated genotype calling and association analysis of SNPs, common copy number 791 polymorphisms and rare CNVs. Nature Genetics. 2008;40(10):1253-60.

792 51. Tam V, Patel N, Turcotte M, Bosse Y, Pare G, Meyre D. Benefits and limitations of 793 genome-wide association studies. Nat Rev Genet. 2019;20(8):467-84.

794 52. Maxim LD, Niebo R, Utell MJ. Screening tests: a review with examples. Inhal Toxicol. 795 2014;26(13):811-28.

796 53. Petros M. Revisiting the Wilson-Jungner criteria: how can supplemental criteria guide 797 public health in the era of genetic screening? Genet Med. 2012;14(1):129-34.

798 54. Michailidou K, Lindstrom S, Dennis J, Beesley J, Hui S, Kar S, et al. Association 799 analysis identifies 65 new breast cancer risk loci. Nature. 2017;551(7678):92-4.

800 55. O'Mara TA, Glubb DM, Amant F, Annibali D, Ashton K, Attia J, et al. Identification of 801 nine new susceptibility loci for endometrial cancer. Nat Commun. 2018;9(1):3166.

802 56. Khera AV, Emdin CA, Drake I, Natarajan P, Bick AG, Cook NR, et al. Genetic Risk, 803 Adherence to a Healthy Lifestyle, and Coronary Disease. N Engl J Med. 2016;375(24):2349-58.

804 57. Tian D, Wang Q, Zhang P, Araki H, Yang S, Kreitman M, et al. Single-nucleotide

805 mutation rate increases close to insertions/deletions in eukaryotes. Nature. 2008;455(7209):105-

8068 .

807 


\section{Figure Legends}

811 Figure 1. A flow-diagram showing the analytical validation framework for detecting and

812 limiting genotyping error in BeadChip array data

814 Figure 2. Aggregate quality control analysis of the GSA data. (A) Principal Component Analysis 815 (PCA) plots of $1 \mathrm{KG}$ data and GSA genotype data. red: African (AFR), yellow-green: Admixed 816 Americans (AMR), dark-green: East Asian (EAS), blue: European (EUR), purple: South Asian

817 (SAS). (B) Heatmaps of BeadChip array quality control analysis of call-rate (left), p10GC

818 (middle), and estimated DNA contamination (right). Color gradient scales for the three panels 819 are as follows: call-rate (orange $<0.94-$ blue $>0.99)$, p10GC (yellow $<0.50-$ blue $>0.60)$ and 820 estimated DNA contamination (rainbow gradient: purple $\sim 1 \%$, blue $\sim 2 \%$, green $\sim 3 \%$, orange /

821 red $\sim$ 4\%). (C) Heatmaps of reproducibility quality control analysis using replicate data as 822 measured by call rate, estimated DNA contamination, number of assays with no genotype calls,

823 and heterozygote to homozygote ratio. Color gradient scales for these four heatmaps are as 824 follows: No genotype calls (blue $<166,000$ - orange $>400,000$ ), and rainbow gradient for call 825 rate (purple $>0.99$ - red $<0.94)$, estimated DNA contamination (purple $<1 \%-r e d>4 \%)$, and 826 heterozygote / homozygote ratio (purple $>2.25-$ red $<1.25$ ) respectively.

828 Figure 3. Three-dimensional scatterplot showing reproducibility of GSA call rate measured in 829 three replicates for each Coriell sample (pairwise analysis of triplicate data). The data is plotted 830 as correlation across triplicates for all measured GSA genotypes for a given DNA sample. Note 831 that most samples had concordance greater than 0.999 between replicates suggesting high 
832 reproducibility. A few samples had off-diagonal points, i.e., those with poor call rates or

833 reproducibility. Color rainbow gradient is from blue $(<0.996)$ to dark red $(1.00)$.

835 Figure 4. Boxplot analysis of the performance metrics of GSA vs 1KG benchmark dataset when 836 assays are classified according to (A) variation type (deletion (DEL), insertion (INS), single

837 nucleotide variant (SNV)), (B) type of single nucleotide change (transition (TNS), transversion

838 (TVS)), (C) frequency of the alternate allele in the 1000 Genomes (1K) data, and (D)

839 interrogation of a low complexity genomic region (microsatellite region (MicroSat),

840 RepeatMasker region (RepMask), or simple repeat (SimRep)). The performance metrics

841 measured and plotted as boxplots for each class / panel are concordance (blue), sensitivity

842 (coral), specificity (green) and positive predictive value (PPV) (orange).

843

844 Figure 5. Bar plot of percentage of GSA assays with a positive predictive value (PPV) <1 as a

845 function of alternate allele frequency bins (allele frequency bins as percentage). The alternate

846 allele frequency bins were defined based on the frequency information in 1000 Genomes (1KG)

847 data.

848

849 Figure 6. Scatter-plot comparison of performance metrics of whole genome sequencing (WGS)

850 and GSA using $1 \mathrm{KG}$ as the benchmark dataset. (A) Scatter plots show sample-level performance

851 metrics of WGS and GSA relative to $1 \mathrm{KG}$ reference data. Plots are concordance (top left; blue),

852 sensitivity (top right; orange), specificity (bottom left; green) and positive predictive value

853 (PPV) (bottom right; maroon) respectively. Each dot represents a single sample's performance

854 metric value. (B) Density scatterplot of each GSA assay's positive predictive value computed 
855 for GSA (y-axis) vs. WGS (x-axis) using $1 \mathrm{KG}$ as the benchmark dataset. Each square represents

856 PPV measured for GSA and WGS relative to $1 \mathrm{KG}$ benchmark dataset, and the color indicates

857 number of assays within each square. Color gradient of each square ranges from 1 assay (dark

858 purple) to 476,828 assays (yellow), therefore, the color on the scatterplot indicates the density of

859 data-points in 2 dimensions.

860

861 Figure 7. Plot of the average percentage of bases within each MAP59 gene covered by whole

862 genome sequencing (WGS) to a read depth of (A) 10x or more (gte10x) (B) 20x or more (gte20x)

863 among the 263 samples. Each WGS nucleotide was required to have a Phred-based quality score

864 of greater than 30 to be considered for this analysis. 
Figures

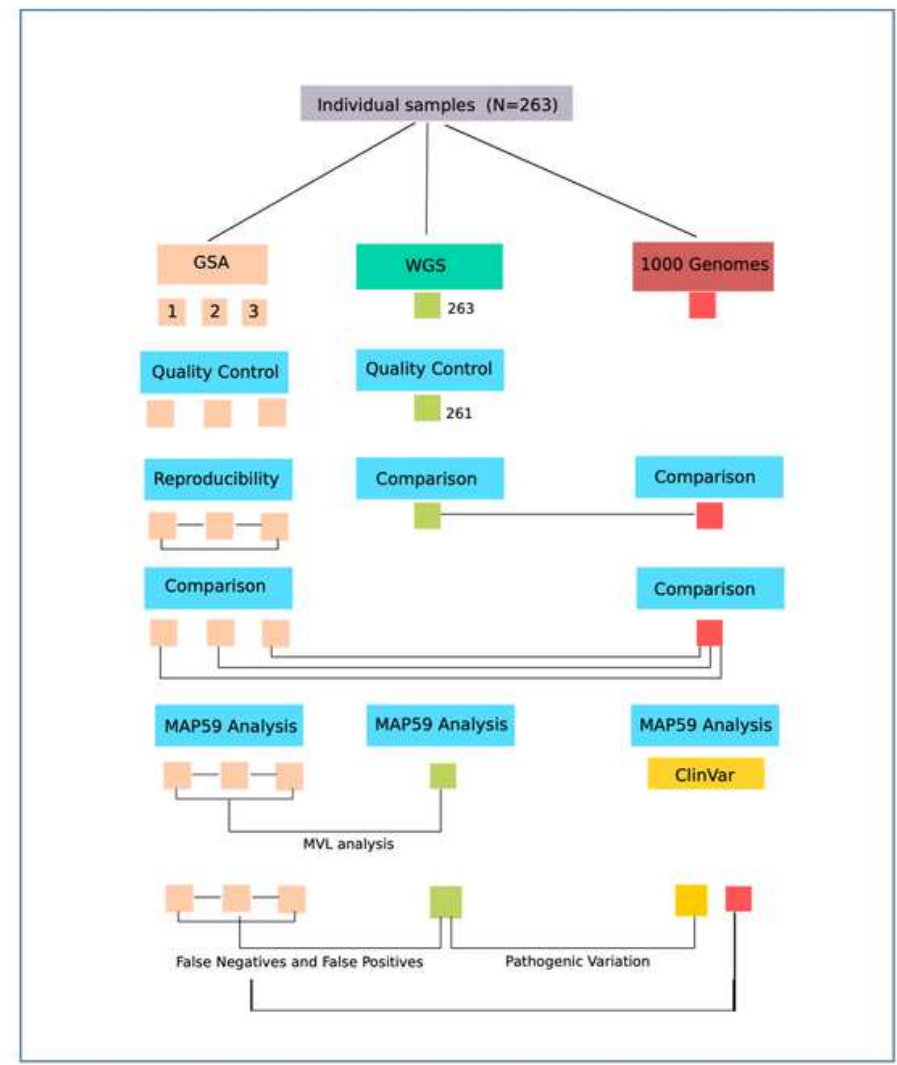

\section{Figure 1}

A flow-diagram showing the analytical validation framework for detecting and limiting genotyping error in BeadChip array data 


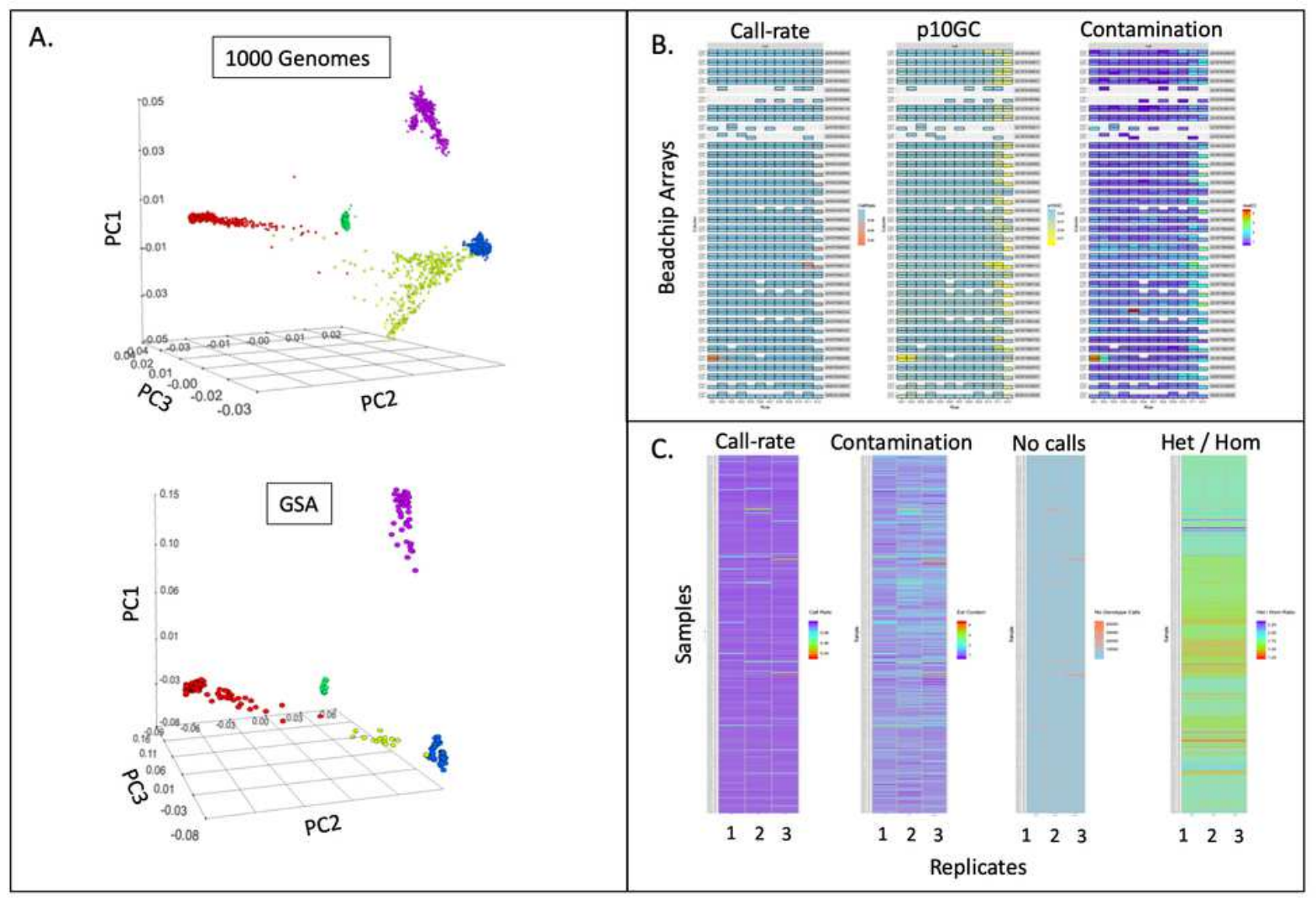

\section{Figure 2}

Aggregate quality control analysis of the GSA data. (A) Principal Component Analysis (PCA) plots of 1KG data and GSA genotype data. red: African (AFR), yellow-green: Admixed Americans (AMR), dark-green: East Asian (EAS), blue: European (EUR), purple: South Asian (SAS). (B) Heatmaps of BeadChip array quality control analysis of call-rate (left), p10GC (middle), and estimated DNA contamination (right). Color gradient scales for the three panels are as follows: call-rate (orange < $0.94-$ blue $>0.99$ ), p10GC (yellow < 0.50 - blue > 0.60) and estimated DNA contamination (rainbow gradient: purple $~ 1 \%$, blue 2\%, green $\sim 3 \%$, orange / red > 4\%). (C) Heatmaps of reproducibility quality control analysis using replicate data as measured by call rate, estimated DNA contamination, number of assays with no genotype calls, and heterozygote to homozygote ratio. Color gradient scales for these four heatmaps are as follows: No genotype calls (blue $<166,000$ - orange $>400,000$ ), and rainbow gradient for call rate (purple $>0.99-$ red $<0.94$ ), estimated DNA contamination (purple $<1 \%-$ red $>4 \%$ ), and heterozygote / homozygote ratio (purple $>2.25-$ red $<1.25$ ) respectively. 


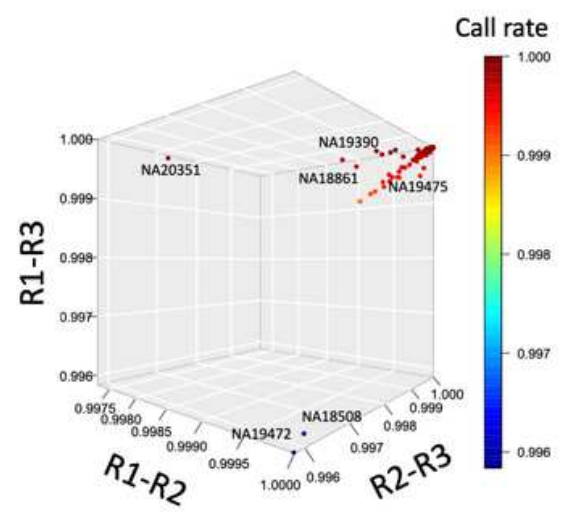

\section{Figure 3}

Three-dimensional scatterplot showing reproducibility of GSA call rate measured in three replicates for each Coriell sample (pairwise analysis of triplicate data). The data is plotted as correlation across triplicates for all measured GSA genotypes for a given DNA sample. Note that most samples had concordance greater than 0.999 between replicates suggesting high reproducibility. A few samples had off-diagonal points, i.e., those with poor call rates or reproducibility. Color rainbow gradient is from blue (< 0.996) to dark red (1.00). 


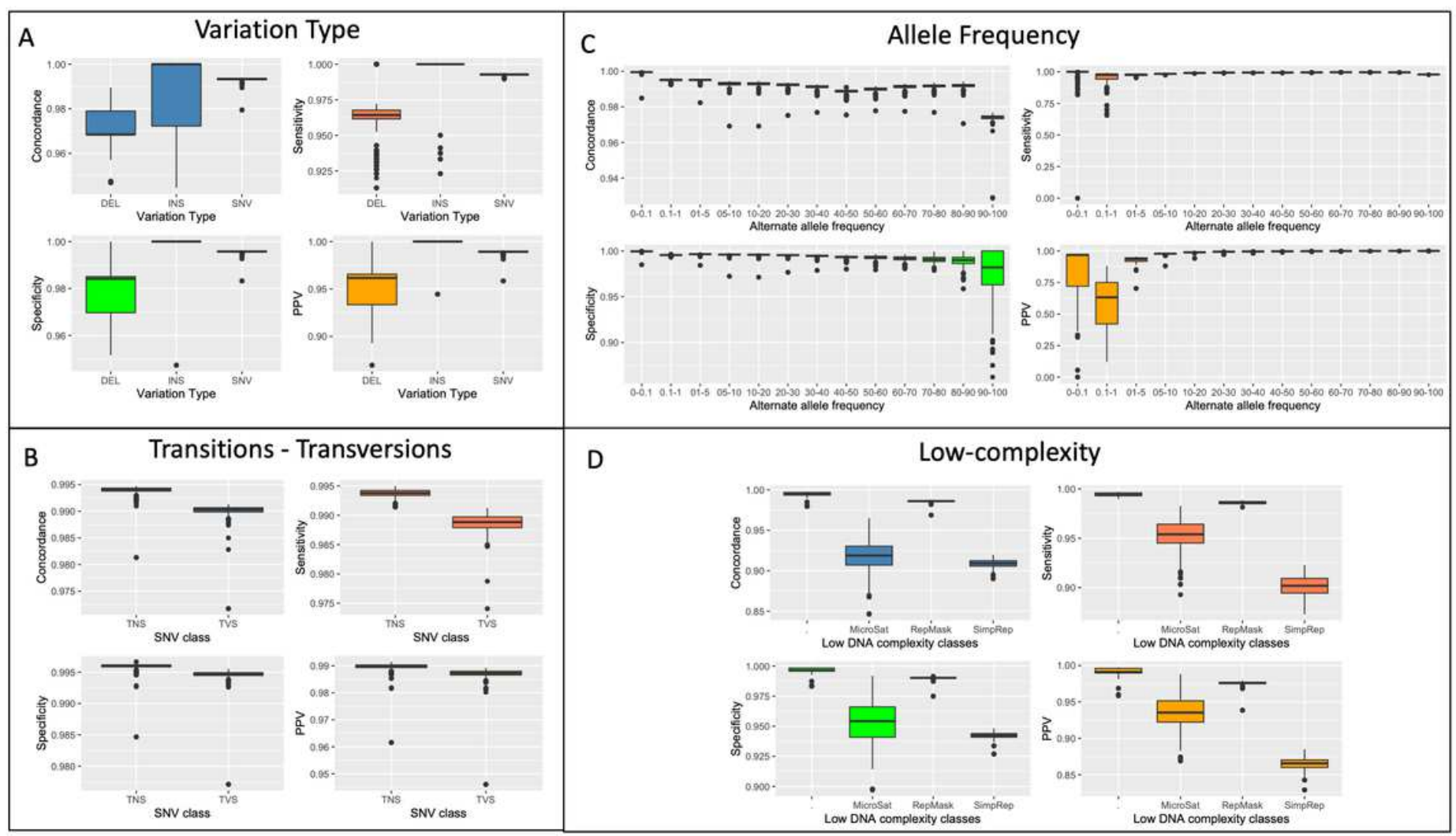

\section{Figure 4}

Boxplot analysis of the performance metrics of GSA vs $1 \mathrm{KG}$ benchmark dataset when assays are classified according to (A) variation type (deletion (DEL), insertion (INS), single nucleotide variant (SNV)), (B) type of single nucleotide change (transition (TNS), transversion (TVS)), (C) frequency of the alternate allele in the 1000 Genomes (1K) data, and (D) interrogation of a low complexity genomic region (microsatellite region (MicroSat), RepeatMasker region (RepMask), or simple repeat (SimRep)). The performance metrics measured and plotted as boxplots for each class / panel are concordance (blue), sensitivity (coral), specificity (green) and positive predictive value (PPV) (orange). 


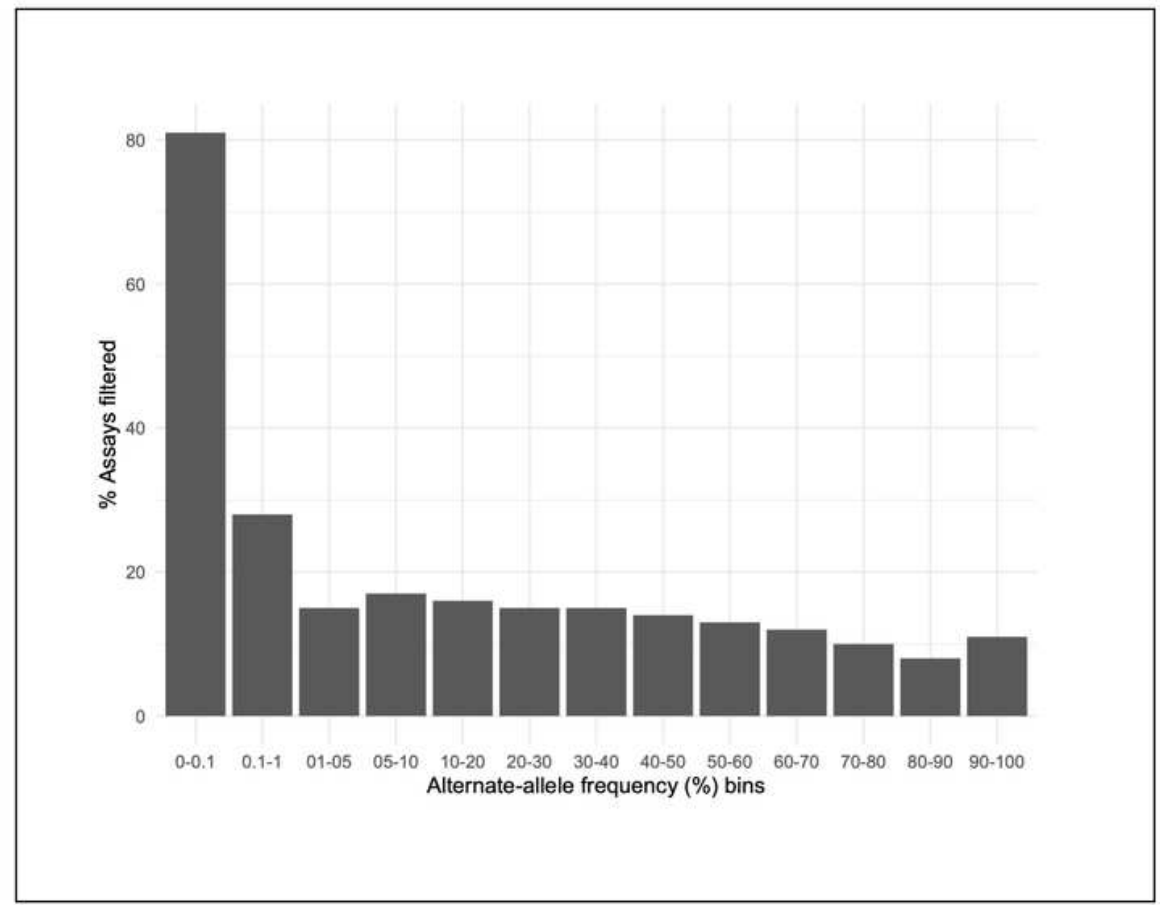

Figure 5

Bar plot of percentage of GSA assays with a positive predictive value (PPV) $<1$ as a function of alternate allele frequency bins (allele frequency bins as percentage). The alternate allele frequency bins were defined based on the frequency information in 1000 Genomes $(1 \mathrm{KG})$ data. 


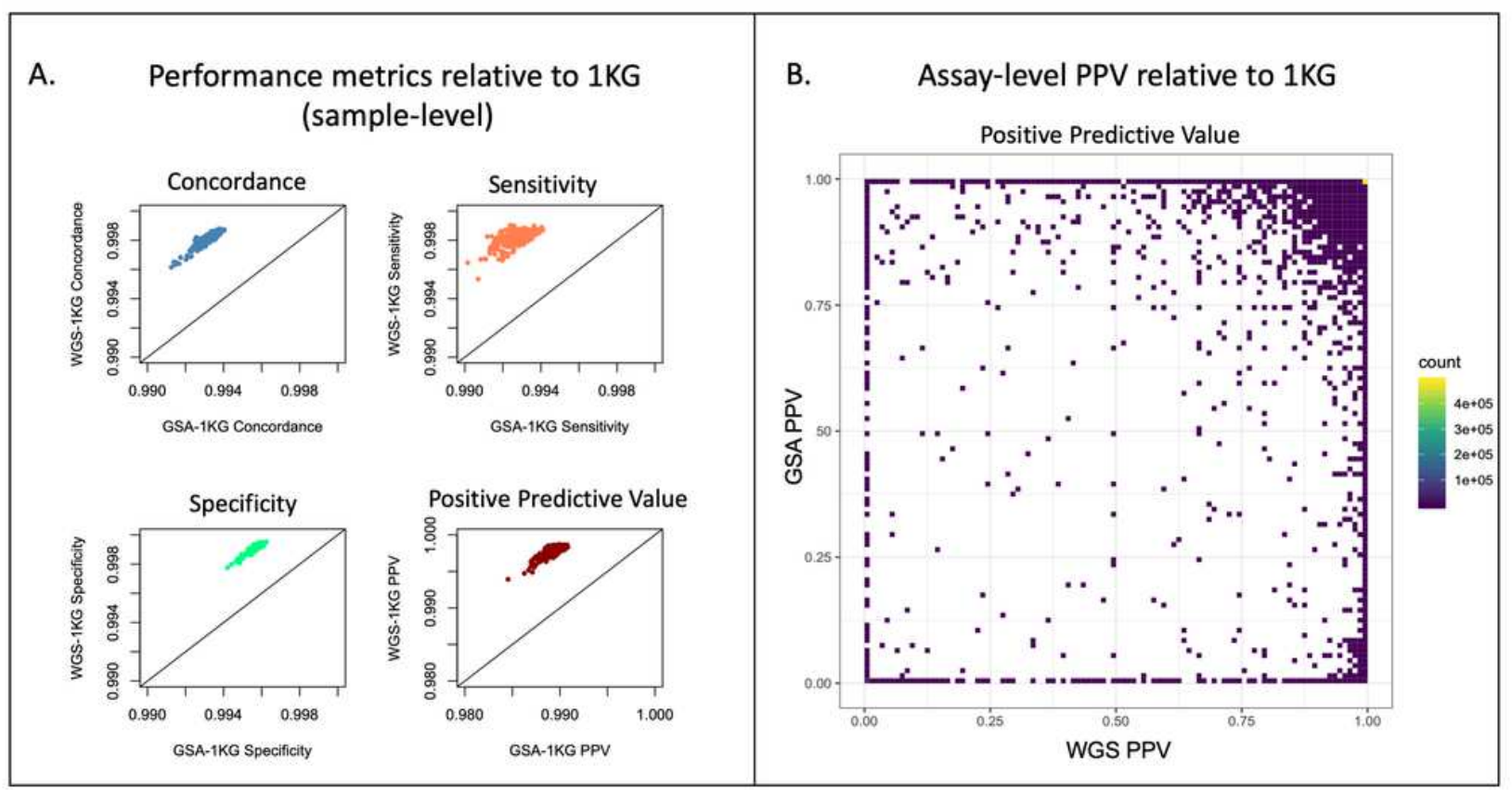

\section{Figure 6}

Scatter-plot comparison of performance metrics of whole genome sequencing (WGS) and GSA using 1KG as the benchmark dataset. (A) Scatter plots show sample-level performance metrics of WGS and GSA relative to $1 \mathrm{KG}$ reference data. Plots are concordance (top left; blue), sensitivity (top right; orange), specificity (bottom left; green) and positive predictive value (PPV) (bottom right; maroon) respectively. Each dot represents a single sample's performance metric value. (B) Density scatterplot of each GSA assay's positive predictive value computed for GSA (y-axis) vs. WGS (x-axis) using $1 \mathrm{KG}$ as the benchmark dataset. Each square represents PPV measured for GSA and WGS relative to $1 \mathrm{KG}$ benchmark dataset, and the color indicates number of assays within each square. Color gradient of each square ranges from 1 assay (dark purple) to 476,828 assays (yellow), therefore, the color on the scatterplot indicates the density of data-points in 2 dimensions. 


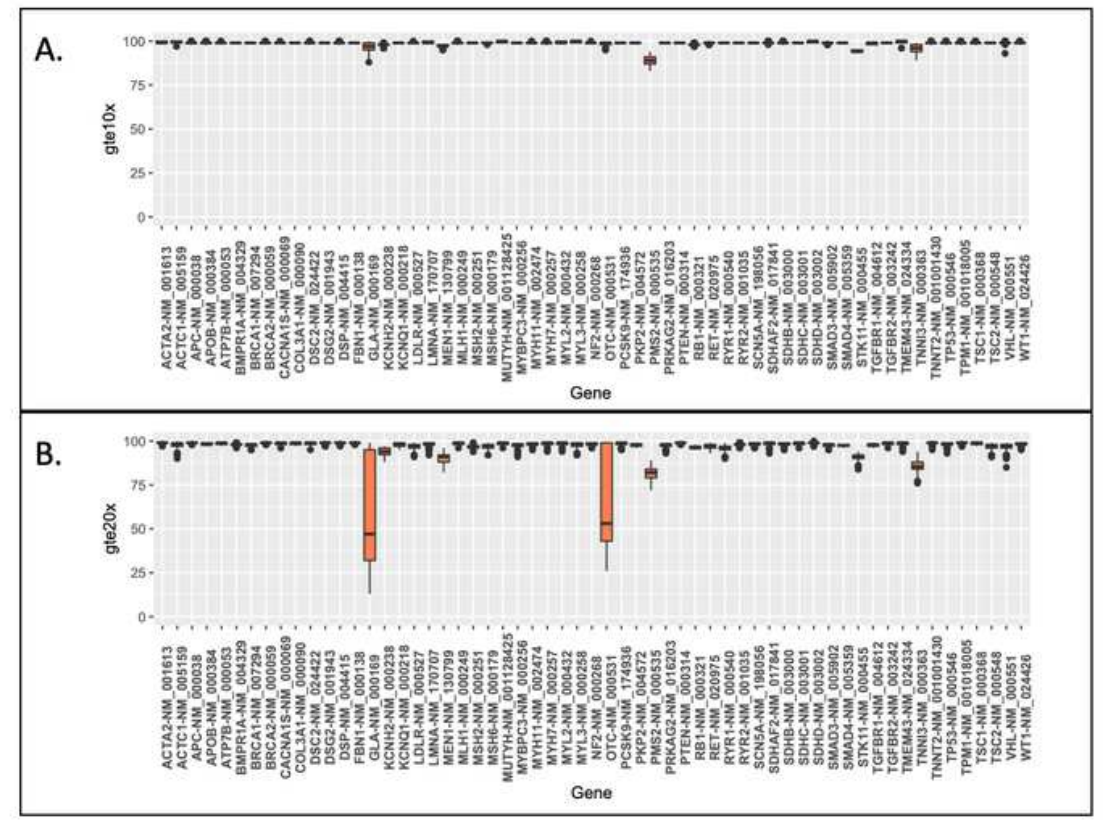

\section{Figure 7}

Plot of the average percentage of bases within each MAP59 gene covered by whole genome sequencing (WGS) to a read depth of (A) 10x or more (gte10x) (B) 20x or more (gte20x) among the 263 samples. Each WGS nucleotide was required to have a Phred-based quality score of greater than 30 to be considered for this analysis.

\section{Supplementary Files}

This is a list of supplementary files associated with this preprint. Click to download.

- SupplementaryMaterialv1.3.docx 